\title{
Research on Ultrasonic Measurement of Partial Discharge Based on Levenberg-Marquardt Algorithm
}

\author{
Yulong Wang ${ }^{1,2, a}$, Lili $\mathrm{Li}^{1,2, a}$ and Xiaoliang Chen ${ }^{2, \text { a }}$ \\ ${ }^{1}$ Key Laboratory of Engineering Dielectrics and Its Application, Ministry of Education, \\ Harbin University of Science and Technology, Harbin 150080, China; \\ ${ }^{2}$ College of Rongcheng, Harbin University of Science and Technology, Rongcheng 264300, China.
}

${ }^{a}$ Fly712@126.com

Keywords: Partial discharge, ultrasonic measurement, Levenberg-Marquardt algorithm, orientation

\begin{abstract}
The core of the detection system is if the partial discharge signal is effective, clear and reasonable during the process of on-line monitoring of partial discharge in cable terminal. The safe operation of cables in power system can be guaranteed through the effective detection method of partial discharge source to predict failure risk of the electrical insulation equipment. The use of ultrasonic method can effectively reduce electromagnetic interference and be easy to achieve on-line monitoring. The partial discharge location can be orientated through changing the sensor position and using Levenberg-Marquardt algorithm. The relationship between magnitude of partial discharge and amplitude of ultrasonic signal, the characteristics and the propagation law of the ultrasonic signal were analyzed in the experiment
\end{abstract}

\section{Introduction}

With the development of micro electronics technology and signal processing technology, especially applications of the piezoelectric transducer element and the integrated component with high sensitivity and low noise in recent years, the sensitivity of ultrasonic measurement for partial discharge is greatly improved. Many researchers [1-3] paid attention to the partial discharge detection in cable terminals in the practical application; however it was rarely paid attention to the detection on partial discharge location in cable terminals using ultrasonic measurements.

T. Pinpart [4] developed a set of unmanned intelligent monitoring system which is based on the partial discharge monitoring system and the insulation condition of high voltage equipment monitoring system using the ultra high frequency method. A. J. Reid [5] in the laboratory completed simultaneously detecting of several partial discharge sources by ultra high frequency method and traditional electrometric method. Wang Wei [6] of North China Electric Power University simulated four kinds of defects using cable joint of $110 \mathrm{kV}$ XLPE power cable customized in Sumitomo Company, which are internal air gap discharge, burr discharge, flashover discharge and suspended discharge respectively. The ultrasonic waveforms of defects were obtained by placing the piezoelectric sensor directly on the outside of cable joints when partial discharge happened, and it is found that the four wave characteristics were different and that energy was mainly concentrated in $50 \sim 250 \mathrm{kHz}$ after discussing time domain and frequency domain characteristics of waveforms

\section{Orientation Theory of Partial Discharge}

Orientation Theory of Partial Discharge. Partial discharge can be seen in IEC60270: 2000 < The Measurement of Partial Discharge >: partial discharge is occurred in the insulation under the high electrical field, which is at the point of lower electrical field. The form of breakdown includes dopants insulation breakdown of gas, partial breakdown within a small range solid or liquid dielectric and local discharge along surface of solid dielectric, and so on. In summary, the condition of partial discharge is determined by electrical field distribution in the insulation device and physical properties 
of the insulation. The location of partial discharge can be calculated by the time gap which is difference between different ultrasonic waves reaching the same position of receivers.

The ultrasonic propagation model of partial discharge is shown in figure 1. Ultrasonic sensors' coordinates are respectively $\mathrm{S}_{1}\left(x_{1}, y_{1}, z_{1}\right), \mathrm{S}_{2}\left(x_{2}, y_{2}, z_{2}\right), \mathrm{S}_{3}\left(x_{3}, y_{3}, z_{3}\right)$ and $\mathrm{S}_{4}\left(x_{4}, y_{4}, z_{4}\right)$ in figure 1.

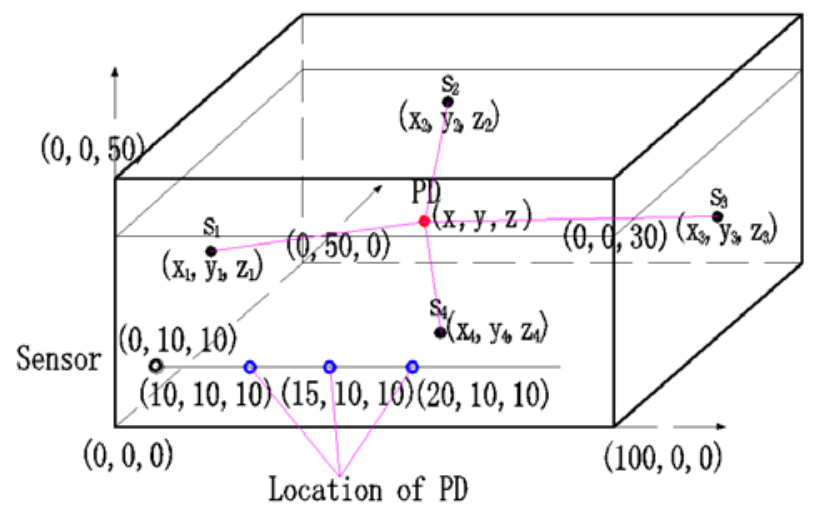

Fig. 1 The ultrasonic propagation model of partial discharge

As shown in figure 1 , if the coordinate of partial discharge position is $(x, y, z)$, the distance between partial discharge position and four ultrasonic sensors $S_{1}, S_{2}, S_{3}$ and $S_{4}$ can be calculated by the coordinates, and the distance can also be calculated by propagation velocity and propagation time of ultrasonic waves. Series of equations are shown in equation (1).

$\left\{\begin{array}{l}\left(x-x_{1}\right)^{2}+\left(y-y_{1}\right)^{2}+\left(z-z_{1}\right)^{2}=\left[V \cdot\left(t_{1}-T\right)\right]^{2} \\ \left(x-x_{2}\right)^{2}+\left(y-y_{2}\right)^{2}+\left(z-z_{2}\right)^{2}=\left[V \cdot\left(t_{2}-T\right)\right]^{2} \\ \left(x-x_{3}\right)^{2}+\left(y-y_{3}\right)^{2}+\left(z-z_{3}\right)^{2}=\left[V \cdot\left(t_{3}-T\right)\right]^{2} \\ \left(x-x_{4}\right)^{2}+\left(y-y_{4}\right)^{2}+\left(z-z_{4}\right)^{2}=\left[V \cdot\left(t_{4}-T\right)\right]^{2}\end{array}\right.$

In the equation, $V$-the propagation speed of ultrasonic wave in the dielectric

$T$-the time of partial discharge

$t_{i}$ - the time arrived at the $i$ th sensor

Among them, $X, Y, Z$ and $T$ are uncertain. The direct solution method is difficult to compute results for nonlinear equations, thus the method of iteration is adopted, as shown in equation (2).

$$
\left\{\begin{array}{l}
f_{1}=\left(x-x_{1}\right)^{2}+\left(y-y_{1}\right)^{2}+\left(z-z_{1}\right)^{2}-\left[V \cdot\left(t_{1}-T\right)\right]^{2} \\
f_{2}=\left(x-x_{2}\right)^{2}+\left(y-y_{2}\right)^{2}+\left(z-z_{2}\right)^{2}-\left[V \cdot\left(t_{2}-T\right)\right]^{2} \\
f_{3}=\left(x-x_{3}\right)^{2}+\left(y-y_{3}\right)^{2}+\left(z-z_{3}\right)^{2}-\left[V \cdot\left(t_{3}-T\right)\right]^{2} \\
f_{4}=\left(x-x_{4}\right)^{2}+\left(y-y_{4}\right)^{2}+\left(z-z_{4}\right)^{2}=\left[V \cdot\left(t_{4}-T\right)\right]^{2}
\end{array}\right.
$$

Therefore the solution of equations is transformed into the solution of least square method, as shown in equation (3). $x, y, z$, and $T$ are determined by calculating equation (3), this is, the location of partial discharge can be ensured.

$$
x^{2}=\left(f_{1}^{2}+f_{2}^{2}+f_{3}^{2}+f_{4}^{2}\right)
$$

The Position Calculation using Levenberg-Marquardt Algorithm. There are many mathematics methods solving nonlinear least square problems, which include gradient descent method, Newton-Gauss iteration method and Levenberg-Marquardt method, and so on. In the article, the Levenberg-Marquardt algorithm was chose to find the multivariable minimum in nonlinear equations.

The Levenberg-Marquardt algorithm can be considered as the combination of the steepest descent method and the Newton-Gauss iterative method. The Levenberg-Marquardt algorithm can be considered as the same as the steepest descent method when the difference between the current value and the true value is large, and the Levenberg-Marquardt algorithm can be considered as the same as the Newton-Gauss iterative method when the difference between the current value and the true value 
is small. When the function $f($.$) and the vector observed x$ are fixed to function $x=f(p)$, the variable $p$ can be calculated. Algorithm steps are as follows:

Step 1. When the initial point $P$ is confirmed, the termination control constant $\varepsilon$ is calculated by the equation (4).

$$
\varepsilon=\left\|x-f\left(p_{0}\right)\right\|
$$

In the equation, $k$ is zero, $\lambda_{0}$ is $10^{-3}$ and $V$ should be greater than 1 . In the paper, 10 was chose.

Step 2. The Jacobi matrix JK is calculated by the equation (5) and the incremental quantity normal equation is constructed to the equation (6) by the Jacobi matrix JK.

$$
\begin{aligned}
& \overline{N_{k}}=J_{k}^{T} J_{k}+\lambda_{k} I \\
& \overline{N_{k}} \cdot \delta_{k}=J_{k}^{T} \varepsilon_{k}
\end{aligned}
$$

Step 3. $\delta_{k}$ is gotten by solving the incremental quantity normal equation. $p_{k}+\delta_{k}$ is equal to $p_{k+1}$ when $\left\|x-f\left(p_{k}+\delta_{k}\right)\right\|$ is less than $\varepsilon_{k}$, thus iteration is stopped and the result is output if $\left\|\delta_{k}\right\|<\varepsilon$. On the contrary, $\lambda_{k} / v$ is equal to $\lambda_{k+1}$ and step 2 is kept on working.

When $\left\|x-f\left(p_{k}+\delta_{k}\right)\right\|$ is greater than or equal $\varepsilon_{k}$, thus $\lambda_{k} \cdot v$ is equal to $\lambda_{k+1}$ and $\delta_{k}$ is gotten by solving the incremental quantity normal equation again, step 1 is kept on working finally.

\section{The Partial Discharge Detection Device by Ultrasonic}

The partial discharge detection device by ultrasonic is shown in figure 2. The needle-plate electrode is used in experiments in this paper and is put into the fuel tank of $50 \times 50 \times 100 \mathrm{~cm}$ in the laboratory, in which the height of the insulating oil is $25 \mathrm{~cm}$. The piezoelectric sensor used in experiments is fixed on the outer wall of tank and the coupling agent is coated on the surface of the sensor and the tank to reduce the attenuation of the ultrasonic signal in the transmission process. The frequency-band range of the ultrasonic signals generated by partial discharge is up to $\mathrm{MHz}$. The energy that is absorbed by sensors is only a small part of the ultrasonic signal energy and the energy that is reflected is only a small part of the ultrasonic signal energy because the ultrasonic signal barging up against tank wall happens to reflection and anacampsis. The sensor which is the frequency band range of 0-400 kHz is selected in the article [7].

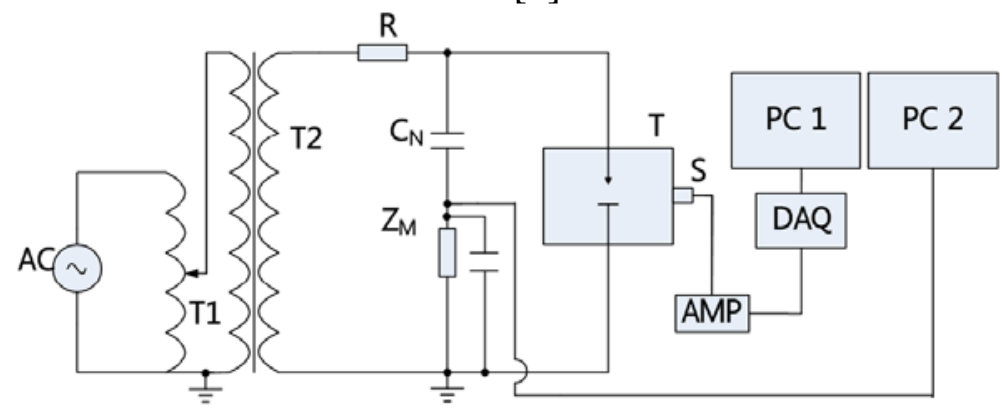

Fig. 2 The partial discharge detection device by ultrasonic

\section{Analysis of Partial Discharge Location by Detection Device}

It is assumed that the position of sensors and the position of the partial discharge source were kept on the same line and that the partial discharge source was measured after moving $5 \mathrm{~cm}$ every time. The time domain waveform and the time domain waveform of Fast Fourier Transform (FFT) in the needle-plate electrode are shown in figure 3(a) and figure 3(b). The ultrasonic signal generated by the partial discharge was due to the release of partial discharge energy in the figure 3, the sampling frequency of the signal is $1 \mathrm{MHz} / \mathrm{s}$, and the threshold of the signal in the experiment is $5 \mathrm{mV}$. It is seen in figure 3 that the signal amplitude increases to $0.01 \mathrm{~V}$ at $0.45 \mathrm{~s}$ suddenly and decreases with the 
increase of time, at the moment, When the frequency reaches 150kHZ, the FFT amplitude increases rapidly and the maximum is up to $6.3 \times 10^{-5}$.

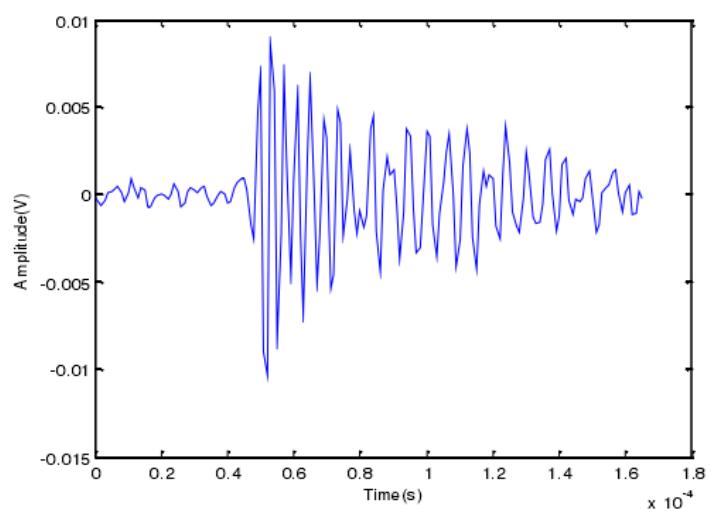

(a) Time domain waveform

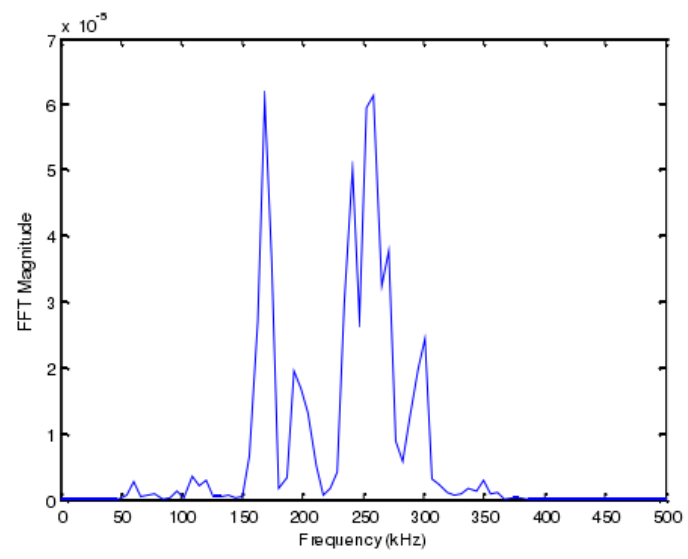

(b) Time domain waveform after FFT Fig. 3 The waveform of partial discharge point $(10,10,10)$ under $6 \mathrm{kV}$

\section{Summary}

The needle-plate electrode is chose in the high voltage equipment during the process of locating the partial discharge source. Although a kind of discharge electrode model was selected, the research method can be applied to other models. The ultrasonic detection of partial discharge was completed in the laboratory and the time domain waveform of partial discharge was collected, finally the partial discharge source was ascertained based on Levenberg-Marquardt algorithm. The result is shown that magnitude of partial discharge is higher in insulation oil; the amplitude of ultrasonic signal is higher, when the threshold of the ultrasonic signal in the experiment is $5 \mathrm{mV}$ based on ultrasonic propagation model of partial discharge.

\section{References}

[1] J. Ramirez Nino, A. Pascacio, Acoustic measuring of partial discharge in power transformers, Measurement Science and Technology. 20 (2009) 2-6.

[2] L. E. Lundgaard, Partial discharge-XIV: Acoustic partial discharge detection-practical application, IEEE Electrical Insulation Magazine. 8 (1992) 36-42.

[3] J. Rubio Serrano, M. V. Rojas Moreno, J. Posada, et al, Electro-acoustic detection, identification and location of partial discharge sources in oil-paper insulation systems, IEEE Transactions on Dielectrics and Electrical Insulation. 19 (2012) 1569-1576.

[4] T. Pinpart, M. D. Judd, Differentiating between partial discharge sources using envelop comparison of ultra-high-frequency signals, IET Science, Measurement, and Technology. 4 (2010) 256-267.

[5] A. J. Reid, M. D. Judd, R. A. Fouracre, et al, Identification of simultaneously active partial discharge sources using combined radio frequency and IEC60270 measurement, IET Science, Measurement, and Technology. 5 (2010) 102-108.

[6] Wei Wang, Xu Cheng, Chong Liu, et al, 2008 International Conference on Condition Monitoring and Diagnosis (Beijing, China, April 21-24,2008). P.1246.

[7] Zhang Jinmei, Investigation on Crystalline Morphology and Tree Discharge Characteristic of PE/MMT Nanocomposites (MS, Harbin University of Science and Technology, China 2009), p.18. 